\title{
Voiding cystourethrogram in the diagnosis of vesicoureteric reflux in children with antenatally diagnosed hydronephrosis
}

\author{
R B Nerli \\ S S Amarkhed \\ I R Ravish \\ Department of Urology, Kles Kidney \\ Foundation, Nehru Nagar, Belgaum, \\ India
}

\begin{abstract}
Prenatal ultrasonography has revolutionized the detection and management of many urological abnormalities. Vesicoureteric reflux (VUR) which develops in $10 \%$ to $15 \%$ of cases of prenatal hydronephrosis, is difficult to predict prenatally. While all children with prenatal hydronephrosis should undergo ultrasonography within the first few weeks of life, there seems to be controversy regarding the role of voiding cystourethrogram (VCUG) in the assessment of these children.
\end{abstract}

Materials and methods: Neonates with antenatally diagnosed unilateral hydronephrosis were prospectively assessed with sonography on day 3-7, and VCUG and isotope imaging at three months.

Results: Seven (16.6\%) children of the 42 children with Society of Fetal Urology grade 0/I/II hydronephrosis on postnatal sonography had evidence of VUR on VCUG. 44.4\% of the refluxing ureters identified involved high grade disease and two $(28.5 \%)$ children required reimplantation.

Conclusions: Children with fetal reflux may be diagnosed prior to urinary tract infection and in whom further renal injury may be prevented. VCUG when performed properly is safe and presents with little risk of infectious and noninfectious complications. VCUG should be done in children in whom hydronephrosis is detected prenatally to restrict the use of VCUG to diagnose VUR. Two patients had infection.

Keywords: antenatal hydronephrosis, voiding cystourethrogram, vesicoureteric reflux

\section{Introduction}

The incidence of reported structural abnormalities detected by antenatal sonography is $1 \% .^{1}$ Nearly $50 \%$ of these anomalies involve the central nervous system, $20 \%$ are genitourinary, $15 \%$ are gastrointestinal, and $8 \%$ are cardiopulmonary. The ability to detect urogenital abnormalities depends on the skill and experience of the sonographer, and usually is better late in gestation, when the fetus is larger and an anomaly is imaged readily. ${ }^{2}$ Hydronephrosis is the urogenital anomaly most often detected, suggesting that an obstructive process may be present. Other etiologies for hydronephrosis include vesicoureteric reflux (VUR), multicystic dysplastic kidney, and certain abnormalities of the ureteropelvic junction and ureterovesical junction. ${ }^{3}$

The controversies involved in the assessment and management of newborns with hydronephrosis continue to challenge clinicians. The widespread use of pre- and postnatal sonography has led to the detection of a large population of newborns with asymptomatic hydronephrosis who require assessment. Most hydronephrosis are detected at 18-20 weeks of gestation, and the prevalence of fetal hydronephrosis, defined as a renal pelvic diameter $>5 \mathrm{~mm}$ is around $2 \%$. Clinically significant hydronephrosis has an estimated incidence of 1 in 600 infants. ${ }^{4,5}$ It is unclear how many of these dilatations represent true obstruction and therefore, require surgical treatment. 
Many cases of hydronephrosis will spontaneously improve or resolve with time postnatally, with no surgical intervention. When an antenatal hydronephrosis is diagnosed, it is imperative to confirm the diagnosis postnatally.

Dilatation of the collecting system may be the result of reflux rather than obstruction. In addition, up to $10 \%$ of patients with pelviureteric junction (PUJ) obstruction also have associated VUR, occasionally severe in degree. Finally, high-grade reflux may lead to secondary PUJ obstruction, wherein the PUJ becomes kinked as a result of severe tortuosity of the refluxing megaureter. ${ }^{6}$ A $15 \%-25 \%$ of neonates with prenatal hydronephrosis will have reflux when checked..$^{7,8}$ Indeed, even if the postnatal ultrasound is normal, a quarter of patients will have reflux. There remains the open question of whether the reflux is clinically relevant. However, patients with antenatal hydronephrosis and sibling reflux are the only two types who may be diagnosed prior to urinary tract infection and in whom further renal injury may be prevented. ${ }^{9}$

We report our prospective study of children with VUR, diagnosed with hydronephrosis on prenatal ultrasonography and evaluated postnatally.

\section{Materials and methods}

Between January 2000 and December 2004, neonates with antenatally diagnosed unilateral hydronephrosis were prospectively assessed at the outpatient center of our hospital. Postnatal ultrasonography was carried on, day 3-7. All ultrasounds were repeated at one month to eliminate understaging secondary to relative dehydration and low urine output in newborns. The severity of hydronephrosis was graded as per the Society for Fetal Urology (SFU) grading system. Neonates diagnosed to have hydronephrosis were put on antibiotic prophylaxis. Ultrasonography was repeated after three months.

Study inclusion criteria were children with SFU grade I/II unilateral antenatally diagnosed hydronephrosis, with no evidence of any other congenital anomaly. Exclusion criteria were sonographic evidence of severe hydronephrosis, bilateral hydronephrosis, hydroureter, duplication, small or echogenic kidney or bladder abnormalities.

All children with prenatally diagnosed hydronephrosis, irrespective of grade of postnatal dilatation underwent voiding cystourethrogram (VCUG) and radioisotope imaging at three months. All children were on antibiotic prophylaxis and received prophylactic antibiotics prior to VCUG. The bladder was drained after VCUG. Children with VUR on VCUG were continued on antibiotic prophylaxis.
Children with no VUR and no obstruction were followed up with no antibiotic prophylaxis. Children with hydronephrosis on ultrasound with no VUR and obstruction on isotope imaging were followed up with three monthly ultrasonography and antibiotic prophylaxis. Children with VUR were advised surgery, if the child developed breakthrough infections during the follow up period.

\section{Results}

During the study period, January 2000 to December 2004, a total of 68 unilateral hydronephrosis were diagnosed on antenatal ultrasonography. Of these 67 neonates (36 males and 31 females) attended the urology out-patient department (OPD) for further evaluation and proposed management within the first week of birth. Postnatal ultrasonography done on day 3-7 revealed 40 neonates had SFU grade I/II dilatation, 25 neonates had SFU grade III/IV dilatation, and 2 children had no evidence of hydronephrosis on sonography.

Forty-two children with SFU grade 0/I/II hydronephrosis on postnatal sonography underwent VCUG and radioisotope imaging at three months. Of these 42 children, seven (16.6\%) had evidence of VUR on VCUG. One of these seven children with VUR had shown no dilatation on postnatal ultrasonography. Of these seven children, 5 were boys and two females. Two children had bilateral VUR whereas the remaining 5 had unilateral reflux. Out of the five males, three underwent prophylactic circumcision.

None of the children undergoing VCUG had urinary tract infection or noninfectious complications. During the follow up period two of these seven children developed pyelonephritis with renal scars inspite of antibiotic prophylaxis. One of these children had unilateral grade IV VUR, and the other had bilateral VUR, left grade V and right grade III. Both patients of bilateral VUR had evidence of reflux nephropathy. The follow up had shown increased size of renal scarring. Both underwent reimplantation in their follow up period. Of the remaining five children, VUR resolved in three children during the follow up period of 18 to 44 months.

\section{Discussion}

Abnormalities of the fetal urinary tract revealed by ultrasonography span a very broad pathological spectrum and are best considered according to severity. They include lethal uropathy, severe nonlethal uropathy resulting in earlyonset renal failure, nonlethal uropathy with a low risk of renal failure, and mild dilatation.

Nonlethal uropathy with a low risk of renal failure accounts for the largest number of infants with prenatally 
Congenital, unilateral, antenatal hydronephrosis

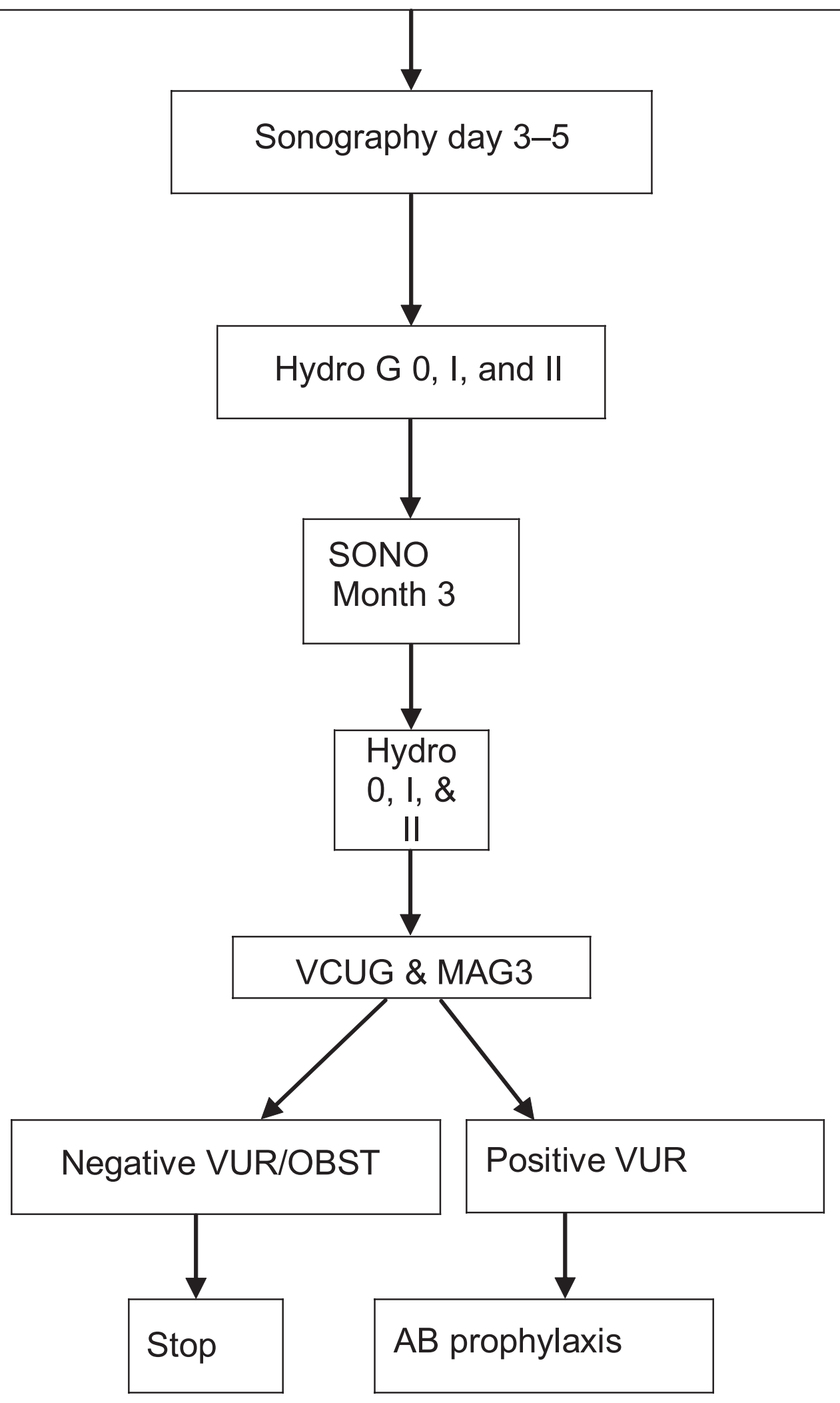

Figure I Algorithm showing assessment and management of VUR.

Abbreviations: $A B$, antibiotic; $V U R$, vesicoureteric reflux; $V C U G$, voiding cystourethrogram. 
detected uropathy referred to urologists. Pyelocaliectasis is the single most common diagnosis. In one study, the contribution made by prenatal diagnosis to the management of 258 children was judged to have been of definite value, defined as revealing clinically unsuspected obstruction or high grade reflux affecting both kidneys or a solitary kidney, in $17 \%$, of probable value (unilateral pathology such as ureteropelvic junction obstruction in infants with a normal contralateral kidney) in 37\%, and of doubtful value (mild dilatation of questionable clinical significance) in $46 \% .{ }^{10}$ Gestational age is an important determinant of diagnostic sensitivity. Hydronephrosis of mild to moderate severity and VUR do not generally give rise to detectable dilatation by the time routine dating or fetal anomaly sonograms are performed in the second trimester.

Measurement of the anteroposterior diameter of the renal pelvis is now routinely performed in most major centers. The grading system advocated by the SFU encompasses the appearances of the collecting system and renal parenchymal thickness.

Centers vary considerably in the extent to which VCUG is routinely employed for the postnatal evaluation of infants with prenatally detected dilatation. Zerin and colleagues reported an overall incidence of VUR of $38 \%$ in patients with prenatal hydronephrosis. ${ }^{9}$ More specifically $25 \%$ of those in whom postnatal ultrasound was normal had reflux. Marra and colleagues prospectively evaluated 47 neonates with mild prenatal hydronephrosis $(0.5$ to $1 \mathrm{~cm}$ pelvic dilatation). All patients underwent a voiding cystourethrogram and reflux was identified in $30 \% .{ }^{11}$ To avoid subjecting large numbers of healthy infants to an invasive investigation, a more selective approach has been suggested, based on ureteric dilatation demonstrated on ultrasonography, either prenatally or postnatally; thick walled bladder or other evidence of outflow obstruction; or caliceal dilatation.

The objections to VCUG being performed in every child with prenatal hydronephrosis has been based on the feeling that VCUG is not an entirely benign procedure due to its invasive nature, radiation exposure, expense and up to a $15 \%$ rate of post-procedure urinary tract infection. ${ }^{12}$ Vates and colleagues retrospectively reviewed the records of all patients diagnosed with prenatal hydronephrosis and who underwent VCUG. ${ }^{13}$ Postnatal VCUG was normal in 138 patients, but it diagnosed bilateral VUR in 15, unilateral VUR in 20, ureterocele in 4 , and refluxing megaureter in 1 . They did not identify any infectious or other complications in infants undergoing VCUG for prenatal hydronephrosis. They believe that VCUG when performed properly is safe and presents little risk in infants.

Sonography is an excellent instrument for screening for abnormalities in the prenatal period, but it is fairly poor for facilitating the diagnosis of VUR. Analysis of the prenatal information identified several factors that are poor indicators of VUR. Female gender had a low correlation with reflux identification. Unilateral prenatal hydronephrosis correlated poorly with postnatal ultrasound findings as well as the collecting system with reflux. Prenatal hydronephrosis correlated with postnatal ipsilateral hydronephrosis in $29 \%$ of the cases and with postnatal ipsilateral VUR in only $33 \% .{ }^{14}$ No specific maternal characteristics were predictive of reflux. Prenatal ultrasound is not the ideal instrument for detecting VUR. Initial postnatal ultrasound was normal in a significant number

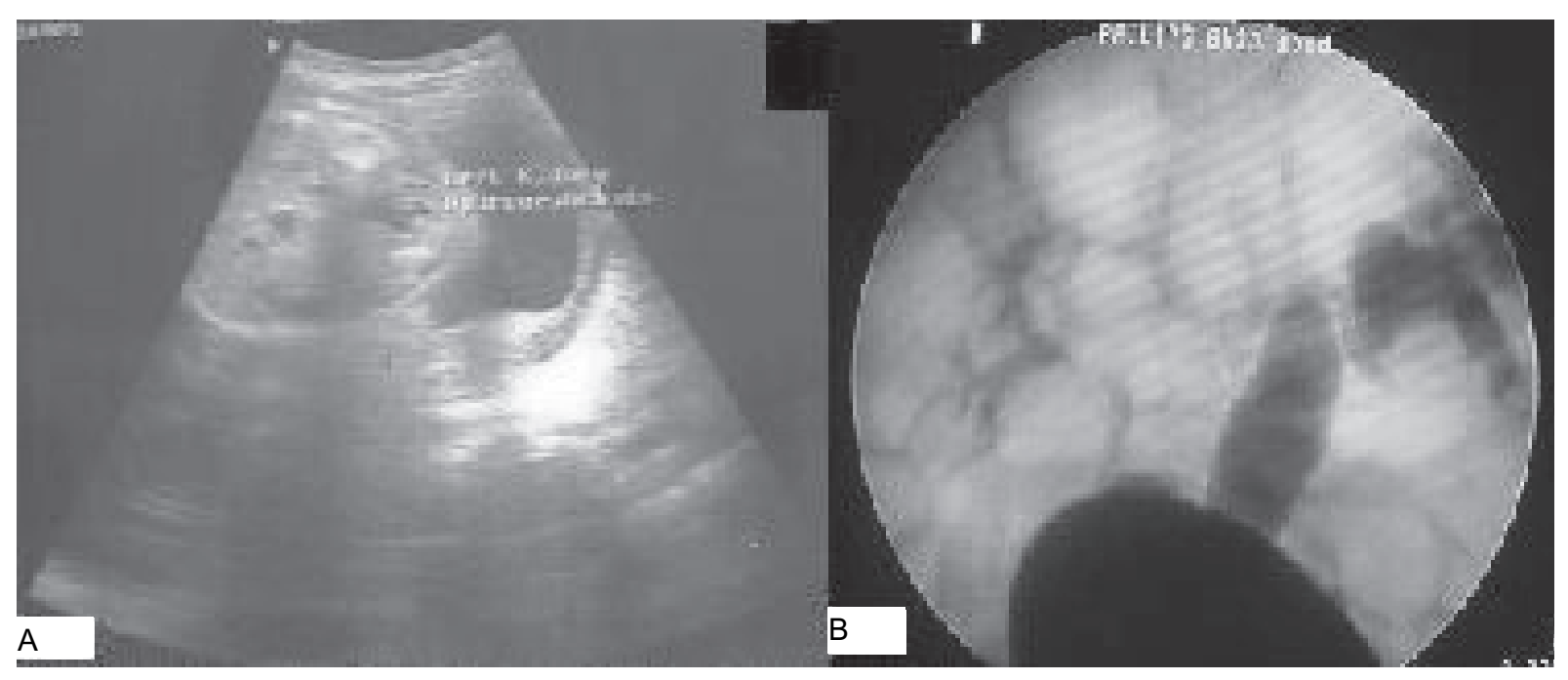

Figure 2 A) postnatal ultrasound shows unilateral hydronephrosis. B) Voiding cystourethrogram shows Rt. grade III and Lt. grade V vesicoureteric reflux. 
of the patients with postnatal VUR. Zerin, ${ }^{9}$ and Najmaldin ${ }^{15}$ noted normal postnatal ultrasound in $18 \%$ to $30 \%$ of patients with VUR. This finding highlights the importance of VCUG irrespective of postnatal ultrasound findings in the cohort of patients with prenatal hydronephrosis. In another cohort of 15 patients, $39 \%$ of the refluxing ureters were identified, involved high-grade disease, and three patients required reimplantation. ${ }^{14}$ This approach of performing VCUG in all patients with prenatal hydronephrosis identifies those in whom postnatal ultrasound is normal and who would otherwise be missed.

In our study (7/42) $16.6 \%$ of children with antenatally diagnosed hydronephrosis had evidence of VUR on VCUG. The incidence of VUR was more in the male children as compared to the female children. $44 \%$ of the refluxing ureters identified involved high grade disease and two (28.5\%) patients required reimplantation. Only 2 patients $(28.5 \%)$ had transient infection which were resolved with antibiotics. In India, where the follow-up ratio is poor due to financial and social reasons, many of these children would have gone undiagnosed and ended up with urinary tract infection, pyelonephritis, renal scars, and loss of renal function, had they not been investigated in detail with VCUG during the evaluation of antenatally diagnosed hydronephrosis.

Khalid Ismail ${ }^{18}$ prospectively followed 264 infants with anternatal renal pelvis dilatation. Two successive neonatal renal ultrasound examinations were performed at day 5 and 1 month after birth. VCUG was performed in all. When both neonatal ultrasound findings were normal (74 infants), the VCUG showed abnormalities in only $5(6.7 \%)$ patients. The sensitivity, specificity, positive predictive value and negative predictive value of two successive ultrasound scans in the neonatal period to predict an abnormality on VCUG were 93\%, 35\%, 33\%, and 93\%, respectively. The authors concluded that routine VCUG does not seem justified.

As more information becomes available, it may be determined that the use of VCUG may be limited by ethnicity or other factors. Until prospective randomized trials are available, not performing VCUG in high-risk patients is probably inappropriate. ${ }^{16,17}$

\section{Disclosure}

The authors report no conflicts of interest in this work.

\section{References}

1. Grisoni FR, Gauderer MW, Wolfson RN, et al. Antenatal ultrasonography: the experience in a high risk perinatal center. J Pediatr Surg. 1986;21:358-61.

2. Fugelseth D, Lindemann R, Sande HA, et al. Prenatal diagnosis of urinary tract anomalies : the valueof two ultrasound examinations. Acta Obstet Gynecol Scand. 1994;73:290-3.

3. Stocks A, Richards D, Frentzen B, et al. Correlation of prenatal renal pelvic anteroposterior diameter with outcome in infancy. $J$ Urol. 1996;155:1050-2.

4. Beetz R, Bökenkamp A, Brandis M, et al. [Diagnostik bei konatalen dilatationen der harnwege.] Urologe A. 2001;40:495-509.

5. Dudley JA, Haworth JM, McGraw ME, et al. Clinical relevance and implications of antenatal hydronephrosis. Arch Dis Child Fetal Neonatal Ed. 1997; 76:31-4.

6. Fung Leo CT, Lakshmanan Y. Anomalies of the renal collecting system: ureteropelvic junction obstruction and infundibular stenosis. In: Belman BA, King LR, Kramer SA, editors. Clinical Pediatric Urology. London, UK: Martin Dunitz; 2002. p. 559-631.

7. Dhillon H. Imaging and follow up of neonatal hydronephrosis. Curr Opin Urol. 1995;5:75-8.

8. Yerkes EB, Adams M, Pope JC, et al. Does every patient with prenatal hydronephrosis need voiding cystogram? J Urol. 1999;162:1218-20.

9. Zerin JM, Ritchey ML, Chang AC. Incidental vesicoureteral reflux in neonates with antenatally detected hydronephrosis and other renal abnormalities. Radiology. 1993;187:157-60.

10. Thomas DFM. Fetal urology and prenatal diagnosis. In: Belman BA, King LR, Kramer SA, editors. Clinical Pediatric Urology. London, UK: Martin Dunitz; 2002. p. 65-81.

11. Marra G, Barbieri G, Moioli C, et al. Mild fetal hydronephrosis indicating vesicourteral reflux. Arch Dis Child. 1994;70:F147-9.

12. Anderson PAM, Rickwood AMK. Features of primary vesicoureteric reflux detected by prenatal sonography. Br J Urol. 1991;67:267-71.

13. Vates TS, Shull MJ, Underberg-Davis SJ, et al. Complications of voiding cystourethrography in the evaluation of infants with prenatally detected hydronephrosis. J Urol. 1999;162(3 Pt 2):1221-3.

14. Anthony Herndon CD, McKenna PH, Kolon TF, et al. A multicenter outcomes analysis of patients with neonatal reflux presenting with prenatal hydronephrosis. J Urol. 1999;162(3 Pt 2):1203-8.

15. Najmaldin A, Burge DM, Atwell JD. Fetal vesicoureteral reflux. Br J Urol. 1990;65:403-6.

16. Gordon AC, Thomas DFM, Arthur RJ, et al. Prenatally diagnosed reflux: a follow-up study. Br J Urol. 1990;65; 407-12.

17. Paltiel HJ, Lebowitz RL. Neonatal hydronephrosis due to primary vesicoureteral reflux: trends in diagnosis and treatment. Radiology. 1989;170(3 Pt 1):787-9

18. Ismailli K, Avni FE, Wissing KM et al. Long term clinical outcome of infants with mild and moderate fetal pyelectasis: validation of neonatal ultrasound as a screening tool to detect significant nephrouropathies. J Pediate. 2004;144:759-65.

19. Ismailli K, Avni FE, Hall M. Results of systematic voiding cystourethrography in infants with antenatally detected renal pelvic dilation. J Pediatr. 2002;141:21-4. 
